\title{
Toward Volume-Based Haptic Collaborative Virtual Environment with Realistic Sensation
}

\author{
Takahide Tanaka, Satoshi Yamaguchi, Lee Jooho, Nobutaka Shimada, Hiromi T. Tanaka \\ Ritsumeikan University \\ Computer Vision Laboratory \\ 1-1-1 Noji-Higashi, Kusatsu, 525-8577, Japan \\ ttanaka@cv.ci.ritsumei.ac.jp
}

\begin{abstract}
In this paper, we propose a volume-based realistic communication system called Haptic Communication that allows participants to interact in real-time with others at remote locations on the network in haptic perception (sense of touch) of soft objects in virtual environments. To provide sense of touch at remote locations in real-time we construct the system as follows. At first virtual soft objects are represented by adaptive volume model in the PCs at the remote locations. Next, from the parameters of positions and forces at contacting points transmitted via network, at each PC the reflection force of the soft object is calculated rapidly and accurately. Eventually, as a result the haptic and visual information are rendered by means of a haptic device PHANToM and a volume graphic software at the PCs. We investigated the efficiency of our system via experiments on a simulation of needle insertion with high force feedback rates at remote locations on a WAN between Ritsumeikan University, Biwako Kusatsu Campus and Osaka University, Toyonaka Campus. The experiment results show that the delay due to network traffic is negligible.
\end{abstract}

Keywords: Realistic-Communication, Telexistence, Haptics, Volume Model, Adaptive-Grid

\section{Introduction}

Virtual reality technology has improved as computers and networks have become faster and more powerful. Some telecommunication systems that allow users to work at remote locations on the network have been developed, and medical and educational applications are expected. This kind of tele-communication we requires visual, auditory and tactile systems. Video chatting is now common. Moreover, force feedback is a tactile way of interacting with virtual objects. It is generated by haptic devices such as PHANToM[10] that can update at $1000 \mathrm{~Hz}$ (more than $1000 \mathrm{~Hz}$ is generally needed as update rate to achieve a real haptic sensation. A high-speed network library developed to transmit force feedback data through a network at $1000 \mathrm{~Hz}$. Thus, the next generation of tele-communication systems need to have the sense of touch.

The VizGrid project[1] is a part of the IT-Program, a five year Japanese national project initiated in 2002. A goal of the project is to create a real-world oriented remote collaboration environment on a grid based on a volume communication concept. The project has enabled the development of volume modeling of output data from simulation or experimental results and a volume modeling of images in the real world by using a multiple-view camera. Hi- kichi et al.[7] proposed a haptic collaboration system without loss of quality of service (QoS). They conducted an experiment to evaluate their system using a rigid and a surface object, and a delay time, packet loss, and information loss were measured. Mortensen et al.[9] presented a study of remote collaboration between people in a shared virtual environment. Two people, one at University College London (UCL) and the other at University of North Carolina Chapel Hill (UNCCH), met together in shared virtual environment and lifted a rigid body object together and moved it to another place. Gunn et al. [6] proposed techniques allowing longdistance sharing of haptic-enabled, dynamic scenes. At the CSIRO Virtual Environments Laboratory, They have successfully used their system to connect prototype of a surgical-simulation application between participants on opposite sides of the world in Sweeden and Australia, over a standard Internet connection. However, previous works did not achieve realistic sensations for the representational model or achieve, real-time performance, and the sense of touch was not well defined. In this study, we constructed our system as follows. First, an adaptive volume model represents virtual soft objects in the PCs at remote locations. Next, each PC calculates the reflection force of the soft object is rapidly and accurately from the parameters of positions and forces at contacting points transmitted via network. Eventually, the haptic and visual information are rendered by a haptic device such as PHANToM and volume graphic software in the PCs. We tested the efficiency of our system via experiments on simulating a needle insertion with high haptic rendering rates at two remote locations on a WAN between Ritsumeikan University, Biwako Kusatsu Campus and Shiga Medical University. The experimental results show that the delay due to network traffic is negligible. Figure 1 presents an overview of the volume-based realistic communication.

\section{System Overview and Architecture}

\subsection{System Overview}

In conventional haptic communication systems, a serverclient is generally used for the surface model, and all data sets such as image information and force information are transmitted[1]. However, transmitting all data sets of volume models for visualizations is difficult because the volume model is huge. We performed a deformation simulation, shown in Figure 2, using the same volume model at a remote location and minimum manipulation parameters to achieve real-time communication. 


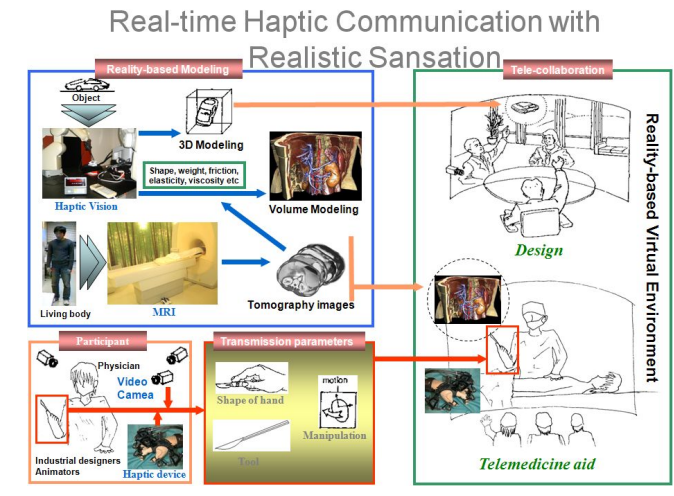

Figure 1: An overview of volume-based realistic communication

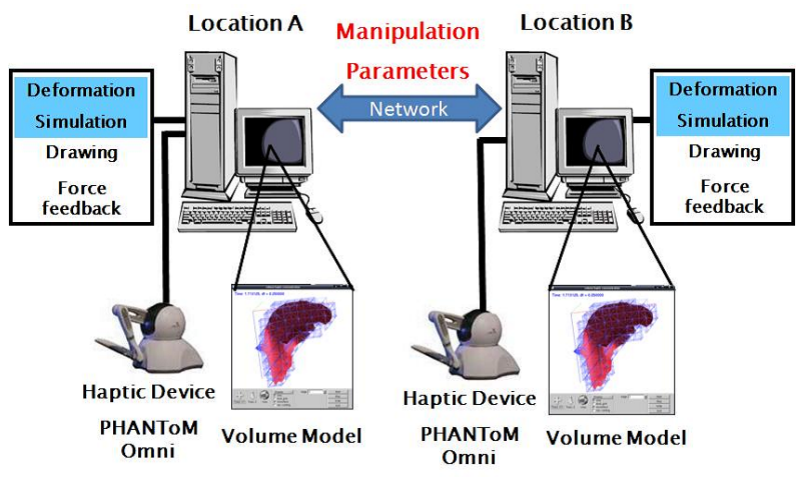

Figure 2: An architecture of system

\subsection{Hardware Architecture}

We used a two desktop PCs Table 1 has the specification of both systems. PHANToM Omni[10] is a haptic device that is connected to both PCs via the IEEE1394 interface. The haptic device enables interaction using the sense of touch a virtual environment with a stylus, which is man-machine interface shaped like a pen.

Table 1: Specification of the PCs

\begin{tabular}{|r|r|r|r|}
\hline & OS & CPU[GHz] & RAM[GB] \\
\hline Server & Windows XP & Intel(R) & \\
& SP2 & Core2 Duo 3.40 & 2.00 \\
\hline Client & Windows XP & Intel(R) & \\
& SP2 & Core2 Duo 2.33 & 2.98 \\
\hline
\end{tabular}

\subsection{Software Architecture}

We used OpenHaptics Toolkit ${ }^{T M}[11]$ for the force feedback. This application enables software developers to add haptic and true 3D navigation to a broad range of applications, from design to games and entertainment to simulation and visualization.

\subsubsection{Haptic Communication Toolkit}

We also used the Haptic Communication Toolkit (HCT)[5] for real-time haptic communication. The HCT is a developer's kit for communication control and also a network library that is developed for communication between two or more haptic devices. However, the HCT can only transmit to 256 bytes to achieve the haptic rate (more than $1000 \mathrm{~Hz}$ ). To go beyond the limitation is difficult because we need a communication band that is faster and proportional to the size of the transmit data. In this study, we achieved realtime haptic tele-communication using the minimum manipulation parameters.

\subsection{Network Architecture}

A server-client type is used in our system. The communication band is 1 giga bit per second on the LAN, and the communication protocol is TCP/IP.

\section{Deformation Simulation Model}

\subsection{Mass-Spring-Damper Model}

The mass-spring-damper (MSD) models called the Voigt model comprising mass node, springs and dampers for representing deformation are widely applied because of the effectiveness in computation and the simplicity in implementation. Figure 3 shows one of an element in the Voigt model. A tetrahedron consists of the six elements and a set of tetrahedron represents a volume model.

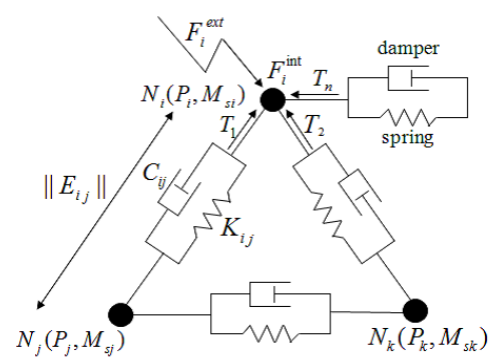

Figure 3: Voigt model

Given a current length $l_{i j}$, a initial length $L_{i j}$, and a directional vector of an edge for $i$ th node, the force value is given as follows:

$$
M_{i} \ddot{P}_{i}=F_{i}+F_{i}^{e x t}
$$

\subsection{Adaptive Volume Modelling}

In our previous work [12], we developed a deformation simulation of a visco-elastic object using the mass-spring dumper model. An input mesh model such as an organ is represented using a binary tree of a set of tetrahedrons without any cracks forming. The model is based on a tetrahedral adaptive mesh for parallel hierarchical tetrahedralization of volume data (Figure 4). The interaction with the virtual object, coordinate, velocity, acceleration, and mass were set to all nodes of the tetrahedral adaptive mesh to simulate the deformation. 


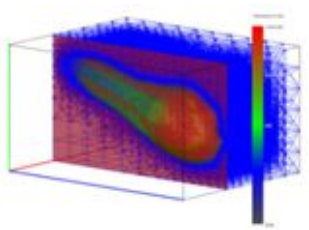

(a) Tetrahedral adaptive mesh

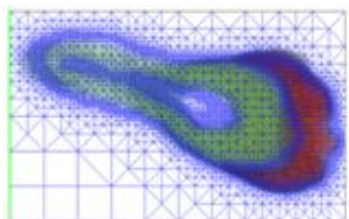

(b) Cross-section view

Figure 4: Tetrahedral adaptive mesh ( A volume model of tooth )

The visco-elastic parameters, elastic parameters and initial length of the edge were set to the edge, consisting of two nodes.

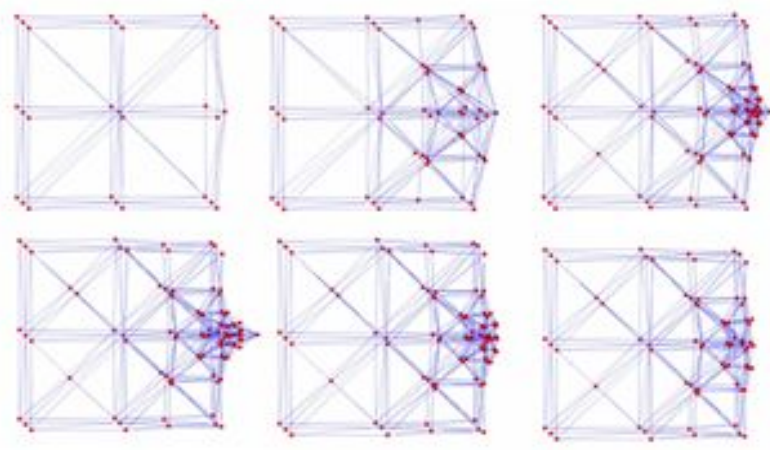

Figure 5: Dynamic tetrahedral adaptive grid of volume data(Manipulation)

Furthermore, we used the dynamic tetrahedral adaptive grid of volume data[13] to simulate deformation of the viscoelastic object rapidly. This algorithm can refine above tetrahedral adaptive mesh interactively. The tetrahedral adaptive mesh is locally subdivided based on the expansion and contraction rate at each edge. The object is constantly represented by specified approximate precision. Figure 5 and Figure 6 show the dynamic tetrahedral adaptive grids of volume data and how they progress. This paper features an extension of this model for manipulation and amotion using the haptic device.

\section{Haptic Communication}

\subsection{Send/Receive Packet}

Manipulation parameters for haptic communication using the above deformation simulation are as follows.

- 3D position of a tip of the stylus in virtual space $(8[$ byte $] \times 3=24[$ byte $])$

- Tetrahedron ID holded by users $(8[$ byte $])$

- $3 \mathrm{D}$ orientation of the stylus $(8[$ byte $] \times 3=24[$ byte $])$

- Node ID manipulated by users (8[byte])

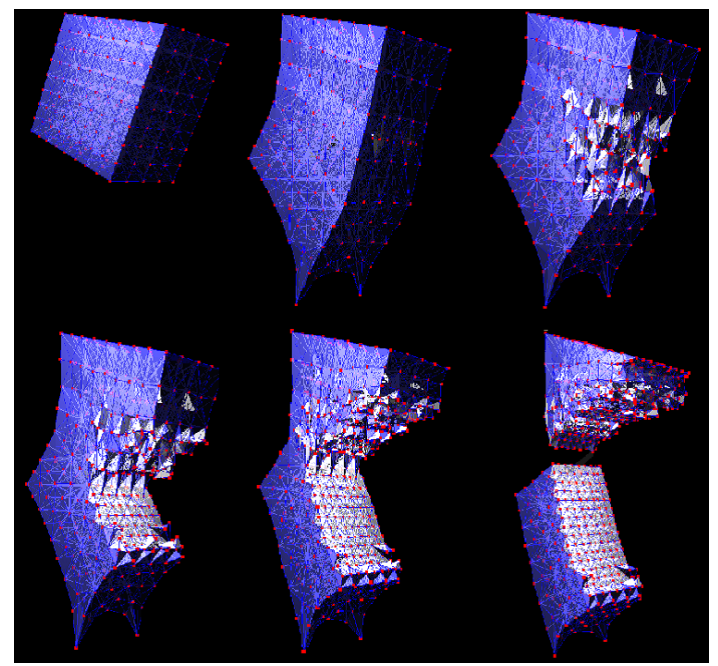

Figure 6: Dynamic tetrahedral adaptive grid of volume data(Amotion)

- $3 \mathrm{D}$ position of Node manipulated by users $(8[$ byte $] \times$ $3=24[$ byte $])$

The manipulation parameters are 72 bytes less than the limitation of the HCT, 256 bytes.

\subsection{Process Flow}

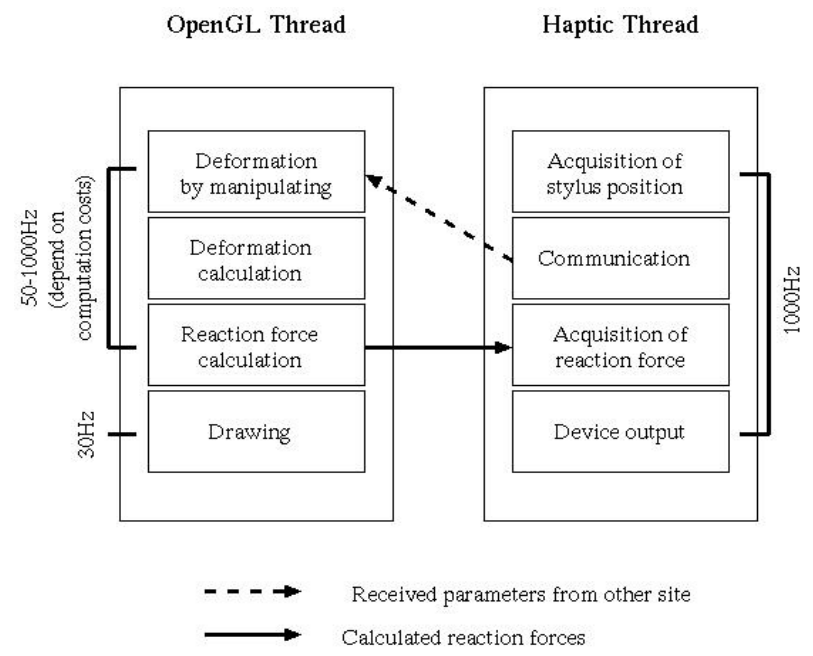

Figure 7: Process Flow

As shown Figure 7, our system has two threads. One is a OpenGL thread and the other is a Haptic thread. the OpenGL thread consists of four processes, a deformation by manipulation, a deformation calculation, a reaction force calculation, and a drawing. An update rate of the OpenGL thread depends on a resolution of the volume model. In this study, a cube of initial level 3 (a number of initial node is 
24 ) is used. This level changes to level 9 ( a number of node is 729) by deformation. Thus, the update rate ranges from $50 \mathrm{~Hz}$ to $1000 \mathrm{~Hz}$ with changing resolution of the volume model. The update rate of the drawing process is controlled as $30 \mathrm{~Hz}$. The haptic thread also consists of four processes, an acquisition of the stylus position, a communication, an acquisition of the reaction force, and a device output. The update rate of the haptic thread is $1000 \mathrm{~Hz}$.

\subsection{Synchronous Method}

To present same deformation simulation to each user, we propose synchronous method. Figure 8 shows an overview of our synchronous method. As shown in Figure 8, a communication between Location A and locationB is not performed directly. Thus, parameters of each location are collected in server and they are sent to each location at the same time. Even if communication time between server and each location are different, deformation process at each location is same because of alignment of parameters in the server.

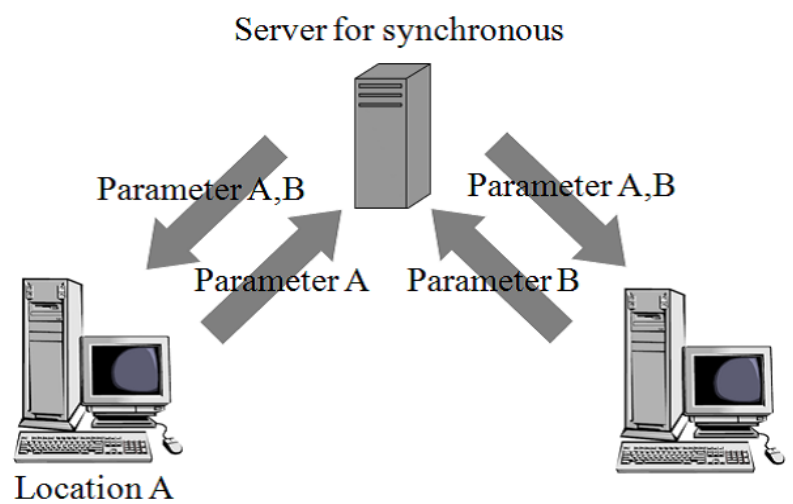

Figure 8: Synchronous Method

\section{Experiment}

\subsection{Communication Time Measurement}

The previous haptic tele-communication system experienced, time delays caused by network traffic and reduced accuracy of force feedback[8]. We performed an experiment on a WAN to evaluate the time delay and force feedback accuracy in our system.

\subsubsection{Exeperimental Condition}

We performed an experiment using remote locations on a WAN between Ritsumeikan University, Biwako- Kusatsu Campus (RU-BKC), and Osaka University, Toyonaka Campus (OU-TC). The distance between the universities on a straight line is about 52 kilometers. The communication time was measured based on the following conditions.

- Conduct experiment using asynchronous method

- Measure the round trip time between server and client

- Measure the number of frames from 10,000 to 25,000
- Calculate average times, maximum times and minimum times

- Repeat five times

\subsubsection{Experimental Result}

Two users performed an interaction of soft objects in a virtual environment. Figure 9 shows a scene of an experiment by two users and Figure 10 shows a representation of the deformation that arises by the interaction. Table 2, Figure 11 and Figure 12 show the experimental results between RU-BKC and OU-TC. Table 2 shows a value of the round trip times of all trials. Figure 11 shows the round trip time on the 1st trial. Figure 12 shows the maximum times, minimum times and average times of all trials. As shown in Table 2, the average times of all trials are about $13[\mathrm{msec}]$. The maximum time of all the trials is $268.7[\mathrm{msec}]$.

Figure 13 shows the round trip time all days once every hour.

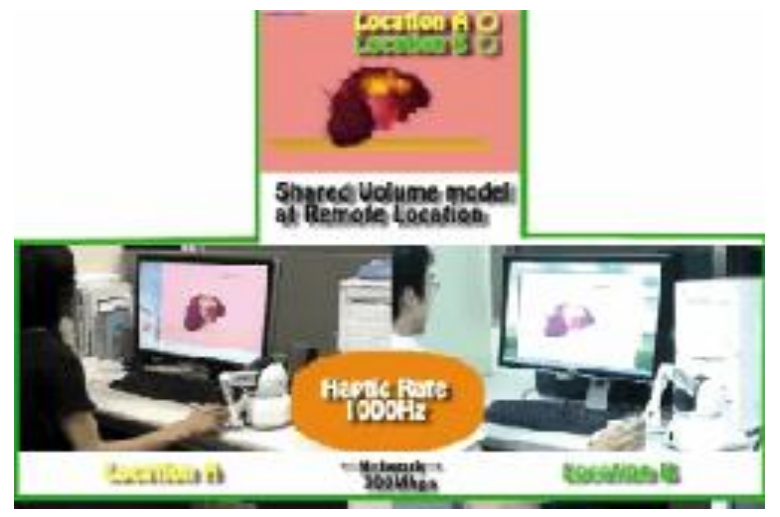

Figure 9: Haptic telecommunication by two users(Scene)

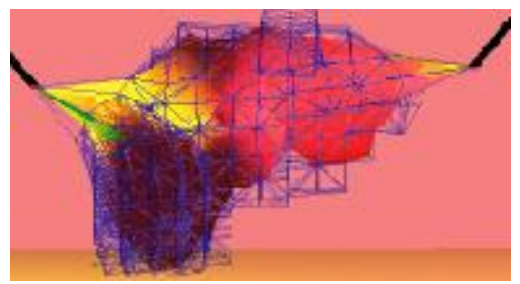

Figure 10: Haptic telecommunication by two users(Display)

Table 2: Round trip times of all trials between RU-BKC and OU-TC [msec]

\begin{tabular}{|c|r|r|r|r|r|}
\hline & 1st & 2nd & 3rd & 4th & 5th \\
\hline \hline Maximums & 53.1 & 57.1 & 57.2 & 268.7 & 87.1 \\
\hline Minimums & 10.9 & 10.9 & 10.9 & 11.0 & 10.9 \\
\hline Averages & 13.1 & 13.2 & 13.2 & 13.6 & 13.1 \\
\hline
\end{tabular}




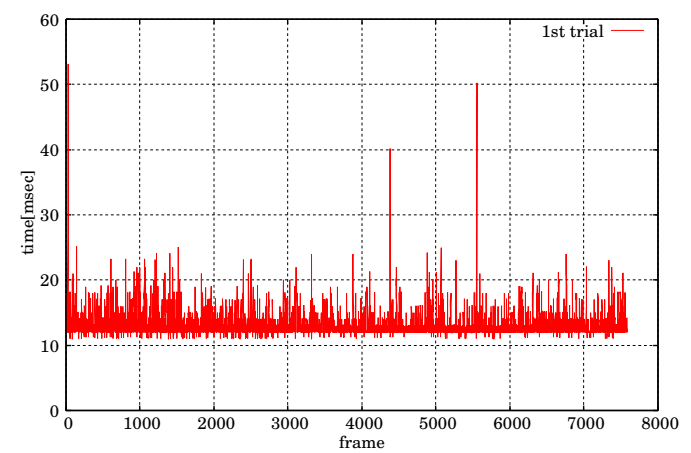

Figure 11: Round trip times on the 1st trial

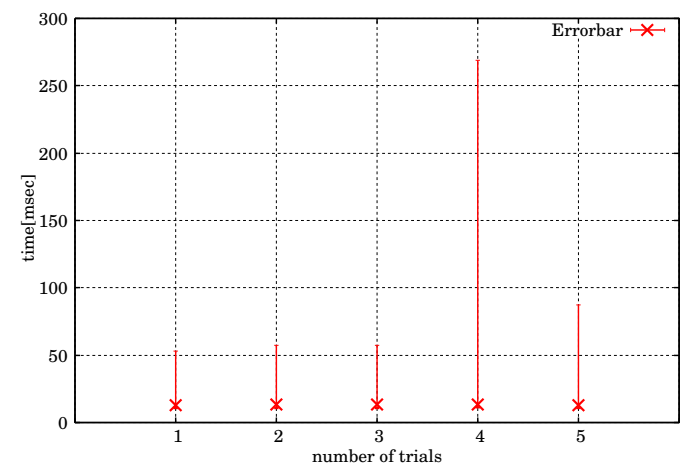

Figure 12: Average times, maximum times and minimum times

\subsubsection{Disscussions}

In any cases, the subjects did not feel any discontinuity of force feedback during while the experiment. The difference between the maximum time and the minimum time was caused by a priority of communications on a WAN. As shown in Figure 12, the average times are almost same as the minimum times. In other words, the communication is almost achieved by the minimum time. All experiment were performed at 10 a.m. As shown in Figure 13, the amount of network traffic at 10 a.m. is about $13[\mathrm{msec}]$. This results give suggestion that the communication performance depends on the running time. Hikichi et al.[7] proposed a haptic collaboration system without loss of quality of service (QoS). They conducted an experiment to evaluate their system using a rigid and a surface object, and a delay time, packet loss, and information loss were measured. Their results show a maximum allowable limit of the delay time is about $80[\mathrm{msec}]$. In this study, the experimental results show the round trip time is totally about 10 to 20 [msec] less than 80 [msec]. Of course, we used a soft object and volume model by different parameters and different experiment procedures in comparison with their experiment. However, in the case of using the soft object, we don' t need high haptic rate such as $1000 \mathrm{~Hz}[2]$.

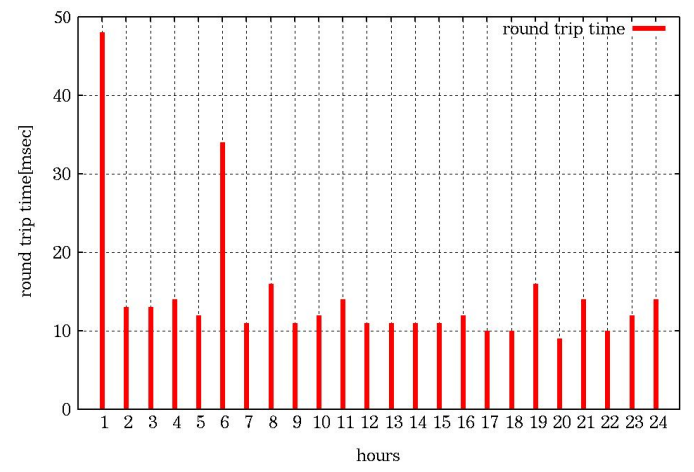

Figure 13: Round trip time all days once every hour

\subsection{Effectiveness of Synchronous Method}

\subsubsection{Experimental Condition}

- Locaion A and Location B are connected to the same LAN

- Use a liver model(Node number:882, Edge number:5299)

- Give a displacement based on a sine function as the input for two nodes

- Control a delay time at about $100[\mathrm{msec}]$.

- Measure a coordinates of four arbitrary points at each location.

- Calculate average differences of coordinates between Location A and Location B. and its standard deviations.

\subsubsection{Experimental Result}

Figure 14 and Figure 15 show the experimental results. Figure 14 shows trajectories of four nodes measured at each location by asynchronous method and Figure 15 shows that of synchronous method.

Table 3 shows average differences of coordinates between Location A and Location B, and Table 4 shows its standard deviations.

In both Figure 14 and Figure 15, solid lines and dot lines represent one of a pair at each location. Solid lines represent trajectories measured at location $\mathrm{A}$, and dot lines are that of location B.

Table 3: Average differences

\begin{tabular}{|c|r|r|r|r|}
\hline & Node1 & Node2 & Node3 & Node4 \\
\hline \hline Asynchronous & 4.88 & -1.16 & 4.35 & -7.01 \\
\hline Synchronous & 4.50 & -0.38 & 3.95 & -3.78 \\
\hline
\end{tabular}

Table 4: Standard Devistions

\begin{tabular}{|c|r|r|r|r|}
\hline & Node1 & Node2 & Node3 & Node4 \\
\hline \hline Asynchronous & 9.46 & 7.86 & 15.70 & 13.84 \\
\hline Synchronous & 5.84 & 4.55 & 4.27 & 6.41 \\
\hline
\end{tabular}




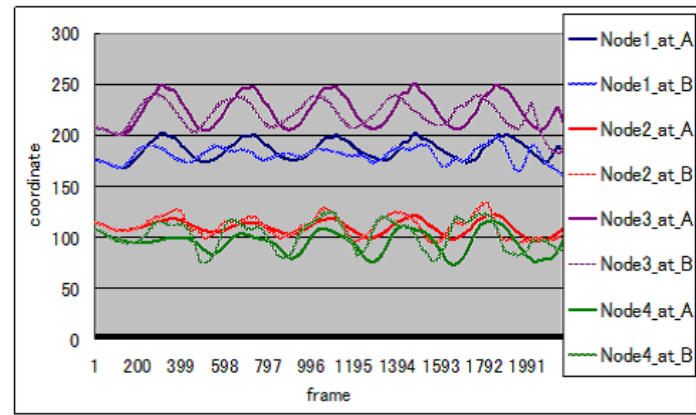

Figure 14: Exprimental Result(asynchronous)

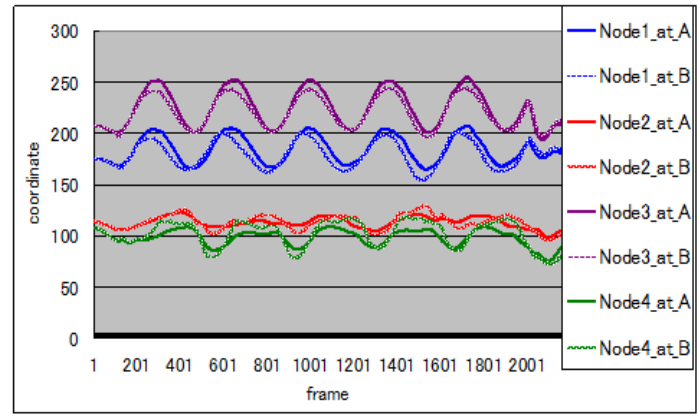

Figure 15: Exprimental Result(synchronous)

\subsubsection{Discussions}

From Figure 14 and Figure 15, trajectory of each pair in synchronous method are more similar than that of asynchronous one. Furthermore, average differences of coordinates between Location A and Location B and its standard deviations are improved by using the synchronous method.

\section{Conclusion}

We described a volume-based haptic communication system that shares an adaptive volume model between remote locations and provides haptic communication to users. Furthermore, we investigated the efficiency of our system via experiments on a simulation of a needle insertion with high haptic rendering rates at two remote locations on a WAN between Ritsumeikan University, Biwako Kusatsu Campus (BKC), and Osaka University, Toyonaka Campus (TC). The experimental results show that the delay due to network traffic is negligible. In future work, we will extend the capability of our system by using multi core CPUs, by synchronizing visualization between the server and client, and by developing an interpolation algorithm for force feedback. We will also perform an experiment by some subjects for subjective assesment.

\section{Acknowlegement}

This research was approved by the Minstry of Internal Affairs and Communications and its strategic telecommunication research and developmental promotion system guideline in 2005. (051307017)(Project Leader:Hiromi T. Tanaka)

\section{References}

[1] VizGrid Project, Final Report of Vizgrid Project. Technical report, Japan, 2007.

[2] C. Basdogan, C.-H. Ho, and M. A. Srinivasan. Virtual environments for medical training: Graphical and haptic simulation of laparoscopic common bile duct exploration. Trans. on Mechatronics, 6(2):269.285, 2001.

[3] G. C. Burdea. Force and Touch Feedback for Virtual Reality. Wiley-Interscience, 1996.

[4] G. C. Burdea and P. Coiffet. Virtual Reality Technology. IEEE, 2003.

[5] I. Goncharenko, M. Svinin, S. Matsumoto, Y. Masui, Y. Kanou, and S. Hosoe. Cooperative control with haptic visualization in shared virtual environments. In Proc. of the 8th Intr. Conf. on Information Visualization, pages 533.538, 2004.

[6] C. Gunn, M. Hutchins, and M. Adcock. Combating latency in haptic collaborative virtual environments. Presence: Teleoperators and Virtual Environments, 14(3):313.328, 2005.

[7] K. Hikichi, H. Morino, I. Fukuda, S. Matsumoto, K. Sezaki, and H. Yasuda. Proposal and evaluation of system for haptics collaboration. Journal of The Institute of Electronics, Information and Communication Engineers, J86-D(2):268. 278, 2003.

[8] J.Arata, H.Takahiro, P.Pitakwatchara, S.Warisawa, K.Konishi, K.Tanoue, S.Ieiri, S.Shimizu, N.Nakashima, K.Okamura, Y.S.Kim, S.M.Kim, J.S.Hahm, M.Hashizume, and M.Mitsuishi. A remote surgery experiment between japan-korea using the minimally invasive surgical system. In Proc. of Intr. Conf. on Robotics and Automation, pages 257.262, 2006.

[9] J. Mortensen, V. Vinayagamoorthy, M. Slater, A. Steed, B. Lok, and M. Whitton. Collaboration in TeleImmersive Environments. In 8th EurographicsWorkshop on Virtual Environments, pages 93.101, 2002.

[10] SensAble Technologies Inc. Phantom OmniTM User ' s Guide, 2004.

[11] SensAble Technologies Inc. OpenHapticsTM Toolkit Ver.2.0 Programmer's Guide, 2005.

[12] Y. Takama, A. Kimura, and H. T. Tanaka. Tetrahedral adaptive mesh for parallel hierarchical tetrahedralization of volume data. Journal of The Information Processing Society of Japan, 48(SIG 9(CVIM 18)):67.78, 2007.

[13] Y. Takama, H. Yamashita, and H. T. Tanaka. Dynamic tetrahedral adaptive mesh generation of volume data. In Symposium on VC/GCAD2007, 2007. 